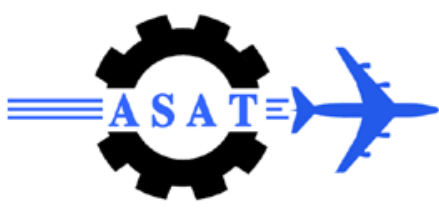

\title{
Optimal Tuning of a PD Controller for a Single-Link Flexible Robot Arm Using Adaptive Genetic Algorithm
}

\author{
Sarah Deif*, Mohammad Tawfik ${ }^{\dagger}$ and Hanan A. Kamal ${ }^{\ddagger}$
}

\begin{abstract}
Flexible manipulators have received wide attention because they are more realistic than their rigid counterparts in many practical conditions. The control of the motion and vibration of an elastic model has inspired many studies in the past decades. In this paper, a finite element model is developed and used for the modeling of the single-link flexible arm. A new adaptive genetic algorithm (AGA) is proposed to optimize the feedback gains of a proportional derivative (PD) controller for the control of the motion and vibration of the flexible arm. The proposed technique is based on a new adaptive mutation operator to adjust the probability of mutation of each chromosome based on the average fitness value at each generation. The results are compared with those obtained by applying simple GA and a direct evaluation of the objective function in the domain. It has been shown that the new developed adaptive mechanism is faster in convergence and the obtained solutions are of higher fitness values. Compared analysis shows the effectiveness of the proposed adaptive GA over traditional genetic algorithms that have the defects of premature convergence and stagnation when applied in optimization problems. Three different objective functions based on minimizing the error function are tested and compared. Multi-objective error fitness function combined with AGA has given the best system response. Simulation results demonstrate the effectiveness of the proposed technique which encourages for further research into the application of the technique in controlling multiple link flexible robots for further applications.
\end{abstract}

Keywords: single-link flexible manipulator, PD controller, simple genetic algorithm, adaptive genetic algorithm, multi-objective optimization

\section{Introduction and Literature Review}

Over the past few decades, the need of high speed manipulation and high payload capability in robot manipulators have drawn a thorough attention to the modeling, control and development of flexible manipulators. Flexible-link robot arms exhibit many advantages over their rigid counterparts including lighter weight, higher speed operation, better maneuverability and lower energy consumption. However, the dynamic analysis of flexible robots and the design of their control system are more challenging problems.

Despite the favorable characteristics, the main disadvantage of flexible robots is the vibration problem due to low stiffness. These oscillations make the precise positioning of the tip of the

\footnotetext{
* Teaching Assistant, Cairo University, sarah_deif@hotmail.com

† Assistant Professor, Cairo University, mohammad.tawfik@gmail.com

‡ Associate Professor, Cairo University, hanan_ak2003@yahoo.com
} 
arm a difficult task. Therefore, the design of the control system must take into account not only the precise angular positioning but also suppressing the vibration developed at the tip due to the high structural flexibility of the manipulator. Several studies have been conducted worldwide in the area of dynamic analysis and control of flexible robot arms presented by the surveys of Dwivedy and Eberhard [1] and Benosman and Vey [2]. These studies have put effort into adopting good control mechanisms that would eliminate structural vibrations.

Many feedback control schemes have been investigated in the literature regarding both the precise positioning and the vibration control of single-link flexible manipulators. Examples include Linear Quadratic Gaussian controllers [3], adaptive control [4], pole placement [5], Lyapunov-based control [6,7], robust control [8], sliding-mode control [9], integral resonant control (IRC) [10] and Regulator-based controllers [11].

Most of these classic or modern controllers assume the exact knowledge of the system dynamics and therefore unsatisfactory for real applications. Therefore, research has been directed towards intelligent-based control such as fuzzy-logic algorithms [12], neural network based techniques [13] and fuzzy neural network controllers (FNN) [14]. However, these methods require complex design methodologies.

Despite the growing research towards studying and designing a sophisticated control algorithm, Proportional-Integral-Derivative (PID) controllers are still widely used in most industrial control applications where PD is a special case of PID. This popularity is due to their structural simplicity, robustness, reliability and broad applicability. The only limitation of PID controllers is the improper choice of PID parameters which may affect the stability of the system.

Genetic Algorithm (GA) has been successfully applied in various research areas such as optimization, design, image processing, machine learning and many other computational intelligence applications [15]. One of the reasons of its broad applicability is that it offers an effective way to search for global solutions. It is also a robust and data-independent search technique. GA was successfully used in tuning the controller parameters for an optimal tip performance of a single-link flexible manipulator $[6,16]$. The main drawback of the conventional GA is that it may converge to a sub-optimal solution. This means it may lead to both slow and premature convergence. Researchers proposed many ideas to solve this problem: all are based on increasing the diversity of the search space. One of these techniques is the concept of adaptive genetic algorithm (AGA).

In AGA, the probabilities of the chromosomal recombination (crossover) and mutation can be adaptive. This has proven to increase the diversity of the search space leading to faster convergence and obtaining better results. Different formulas of adaptive operators were investigated over the years where the probabilities of crossover and mutation were represented as a function of the fitness values of the solutions, the average fitness and the maximum fitness at each generation $[17,18]$.

This work presents a new adaptive genetic algorithm technique that is used to tune the feedback gains of the PD controller which is designed to control the hub angle position and the vibration developed on the single-link flexible manipulator. The dynamics of the flexible arm is derived using the Hamilton's principle and modeled using the finite element method (FEM). Three different objective functions are introduced and compared (HAISE, TDISE and ADISE). Furthermore, the proposed technique is compared with the simple GA. As far as this survey is concerned, AGA hasn't been applied before in the optimization of the controller gains for single-link flexible manipulators. 


\section{The Single-Link Flexible Manipulator Model}

Using Hamilton's principle:

$$
\int_{t_{1}}^{t_{2}} \delta(U-T-W) d t=0
$$

where $U$ is the potential energy, $T$ is the kinetic energy, and $W$ is the work done by the external forces.

The potential energy of the beam moving in a horizontal plane may be written as:

$$
U=\frac{1}{2} \int E I\left(\frac{d^{2} w}{d x^{2}}\right)^{2} d x
$$

where $E I$ is the beam bending stiffness and $w$ is the displacement of the beam measured from the rigid body line as shown in Fig. 1

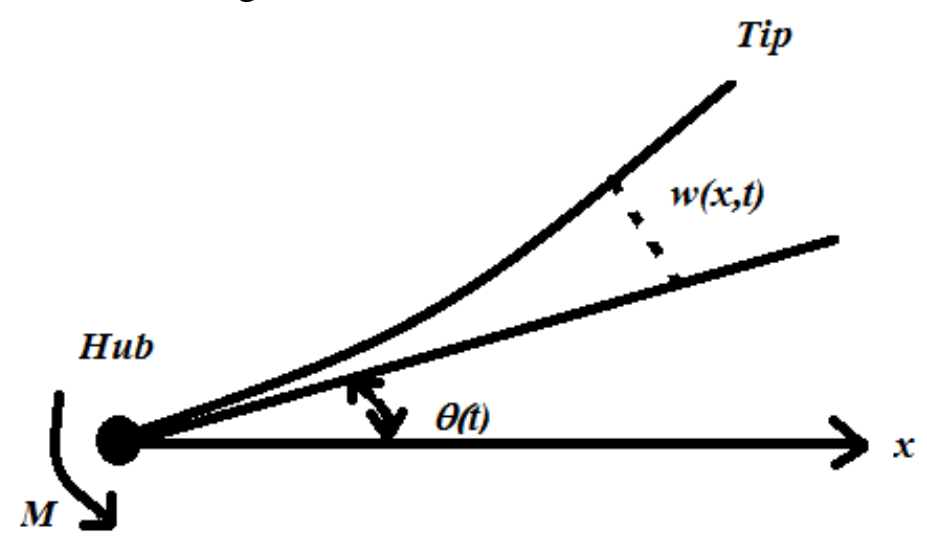

Fig. 1. A single-link flexible robot arm

Meanwhile, the kinetic energy may be written as:

$$
T=\frac{1}{2} \int \rho A\left(\dot{\theta}^{2} x^{2}+2 \dot{\theta} \dot{w} x+\dot{w}^{2}\right) d x
$$

where $\theta$ is the angular displacement of the rigid beam measured from the x-axis. The external work done by the servo motor may be written as:

$$
W=M \theta
$$

where $M$ is the moment provided by the motor

Using the standard finite element modeling techniques, we may write the displacement of any element in the form:

$$
w(x, t)=\left[\begin{array}{llll}
N_{1}(x) & N_{2}(x) & N_{3}(x) & N_{4}(x)
\end{array}\right]\left\{\begin{array}{c}
w_{1}(t) \\
w_{1}^{\prime}(t) \\
w_{2}(t) \\
w_{2}^{\prime}(t)
\end{array}\right\}
$$

where $N_{i}$ is the shape function, and $w_{i}$ and $w_{i}^{\prime}$ are the nodal displacement and the slope respectively. The above equation may be written in compact form as:

$$
w(x, t)=\lfloor N(x)\rfloor\{\Delta(t)\}
$$


Applying Hamilton's principle and using the above equation, we may write the equation of motion as:

$$
\left[\begin{array}{cc}
M_{B} & M_{t} \\
M_{t} & J
\end{array}\right]\left[\begin{array}{c}
\ddot{W} \\
\ddot{\theta}
\end{array}\right]+\left[\begin{array}{cc}
K_{B} & 0 \\
0 & 0
\end{array}\right]\left[\begin{array}{l}
W \\
\theta
\end{array}\right]=\left[\begin{array}{c}
0 \\
M
\end{array}\right]
$$

where $M_{B}$ is the assembled mass matrix, $M_{t}$ is the assembled coupling mass matrix, $J$ is the rigid beam mass moment of inertia around the hub and $K_{B}$ is the assembled bending stiffness matrix.

On the other hand, the element mass matrix may be written as $\int_{x_{1}}^{x_{2}} \rho\lfloor N(x)\rfloor\{N(x)\} d x$ where $\rho$ is the beam mass per unit length, the element coupling mass matrix $\int_{x_{1}}^{x_{2}} \rho x\lfloor N(x)\rfloor d x$, the element bending stiffness matrix $\int_{x_{1}}^{x_{2}} E I\left[N_{x x}(x)\right]\left\{N_{x x}(x)\right\} d x$ and the beam moment of inertia is $\rho l^{3} / 3$ where $l$ is the element length.

The time integration has been performed using the Newmark method which approximates the time response using the algorithm in (19).

\section{The Proposed Genetic Algorithm}

The basic principles of genetic algorithm (GA) were first proposed by John Holland [20]. GA uses operations found in natural genetics based on the Darwinian Theory. GA constitutes stochastic search methods that have been used in a wide spectrum of applications such as control systems engineering and robotics [21].

In the beginning, a random population of chromosomes of size $N_{\text {pop }}$ is generated to represent the PD gain values $\left(k_{p}, k_{d}\right)$ in the given bounds. A real-coded GA [22] is used to represent the values of the genes. Then, these values are applied to the flexible manipulator system and a corresponding fitness value is calculated for each chromosome by evaluating the fitness function (in this paper three objective functions are deployed which will be discussed in detail later: HAISE, TDISE and ADISE). The choice of the fitness function plays an important role to distinguish between the most "fit" and the less "fit" solutions of a given problem. Based on the fitness value of each individual, a group of chromosomes are selected to propagate into the next generation. The higher the fitness the more probable it will survive. The selected chromosomes are then mated together to recombine the best features of the parent chromosomes to produce better offspring. A mutation operator is then introduced with a small probability to ensure diversity in the search space. The process continues until a maximum number of generations $G_{\max }$ is reached: which is the stopping criterion utilized. The fittest chromosome of the last generation is the solution of the optimization problem.

\subsection{Genetic Operators}

Three genetic operators are applied to the current population in order to create a new generation of chromosomes of the same population size. These operators are reproduction, crossover and mutation respectively. The choice of both the crossover and mutation operators is of a great importance and can determine the performance of the whole genetic algorithm. By using different probabilities of both operators, the speed of convergence and the accuracy of the solution can be controlled. Two parent chromosomes are selected from the mating pool then undergo genetic operations until a new generation of the same size $N_{\text {pop }}$ is created. 


\subsubsection{Reproduction}

The reproduction operator is the element that performs the "Survival of the Fittest" or natural selection function in a GA. "Roulette Wheel Selection" method is used [21]. Power-law fitness scaling is performed prior to the selection process in order to avoid premature convergence.

\subsubsection{Crossover}

The crossover operator recombines genetic information from the two selected parents to produce offspring with characteristics from both at a probability of crossover $P_{c}$. An arithmetic crossover operator for real (decimal) numbers called weighted average operator [6] is used.

\subsubsection{New Added Mutation Value}

The drawback of the conventional GA regarding mutation is that the chromosomes are mutated by random values in the search space diverting the solution away from the optimum solution which leads to both slow and premature convergence. This new proposed technique is based on mutating the chromosomes with values in the vicinity of the "good featured" chromosomes.

A selected individual gene value is replaced with $X$ according to the probability of mutation as described below:

$$
X=r_{1}+\left(r_{2} *\left(k_{\text {best }}-r_{1}\right)\right)
$$

where $r_{1}$ is a random number that lies in the bound of the feedback gain

$r_{2}$ is a random value in the range $(0,1)$

$k_{\text {best }}$ is the feedback gain of the "fittest" chromosome in this generation.

First, a random number in the bound of the gain values is selected $r_{1}$ then a random mutation value is added that makes $r_{1}$ gets closer to the vicinity of $k_{\text {best }}$. This proposed technique adds diversity to the search space as it increases the area of this neighborhood.

\subsubsection{New Adaptive Mutation Operator}

An adaptive probability of mutation is proposed to realize the two goals of maintaining diversity in the search space and sustaining the convergence capacity of the GA which is defined as:

$$
P_{m}=\alpha+\left(\beta *\left(1-f_{\text {avg }}\right)\right)
$$

where $\alpha$ and $\beta$ are constant parameters determined by simulation trials

$f_{\text {avg }}$ is the average fitness value of a population that lies in the range $(0,1)$

In adaptive GA, the mutation probability depends on the average fitness of the population. At first few generations, $f_{\text {avg }}$ is a small value as the solution is still far away from the optimal one. Therefore, $P_{m}$ is high with a maximum value of $(\alpha+\beta)$ resulting in new chromosomes introduced to the search space and the 'disruption' of the sub-average fitness solutions. The average fitness then increases as the GA successfully moves towards a better solution and therefore $P_{m}$ is decreased until it reaches a minimum value of $\alpha$ when $f_{\text {avg }}$ reaches its highest value. This means that the high-fitness solutions are 'protected' and maintained through generations. Several simulation trials have been carried out resulting in the values $\alpha=0.05$ and $\beta=0.1 \sim 0.15$ that proved high performance of the proposed technique. 


\subsection{Evaluation Function}

The most crucial step in applying GA is to choose the objective functions that are used to evaluate the fitness of each chromosome. In this paper, the objective of the optimization problem is to obtain better performance for the control of the angular position and the vibration of the single-link flexible robot, i.e. to obtain optimum values of the feedback gains with which the PD controller can drive the hub angle and the tip deflection to predefined values as fast as possible with minimal oscillation. This is achieved by minimizing the fitness (error) function. In this paper, three different objective functions (HAISE, TDISE and ADISE) are defined based on the Integral of the Squared Error (ISE) where $I S E=\int_{0}^{T} e(t)^{2} d t$ and $e(t)$ is the output error function to be minimized.

Further, a transformation is clearly required because low fitness values are much more clearly distinguished than high fitness values. The objective function value of any chromosome $g_{i}$ is simply transformed to a fitness function value $f_{i}$ that lies in the range of $(0,1)$ which is described as:

$$
f_{i}=\frac{1}{\left(1+g_{\max }-g_{i}\right)}
$$

Where $g_{i}$ is the objective function of any chromosome $i$

$g_{\max }$ is the best value for the objective function at each generation

$f_{i}$ is the corresponding fitness function of any chromosome $i$

\subsubsection{HAISE}

The first objective function (Hub-Angle Integral of the Squared Error) is based on the minimization of the output error $e(t)$ which is equal to the difference between the feedback hub angle $\theta(t)$ of the system and the input predefined set point angle value which is set to zero.

$$
\text { HAISE }=\int_{0}^{T} \theta_{h u b}(t)^{2} d t
$$

\subsubsection{TDISE}

The second objective function (Tip-Deflection Integral of the Squared Error) is similar to HAISE but with the tip deflection $w_{\text {tip }}$ replacing the hub angle $\theta_{\text {hub }}$.

$$
T D I S E=\int_{0}^{T} w_{t i p}(t)^{2} d t
$$

\subsubsection{ADISE}

A multi-objective function (Angle and Deflection Integral of the Squared Error) based on the simple "sum of the weighted cost functions" is introduced [23].

$$
A D I S E=\left(w_{1} * H A I S E\right)+\left(w_{2} * T D I S E\right)
$$

where $w_{1}$ and $w_{2}$ are weighting factors

The key to this method is to determine the values of the weighting factors. In this paper, values of the minimized cost functions (HAISE and TDISE) are first evaluated and used as the weighting factors. Then, the weighting factors are set to $w_{1}=\min (T D I S E)$ and $w_{2}=$ min $(H A I S E)$. This simple method is deployed to make sure that the "weighted cost functions" lie in the same range of values. 


\section{The PD Controller}

An advanced auto tuning Proportional Derivative (PD) feedback controller is presented as shown in Fig. 2 for the flexible manipulator plant to search optimal feedback gain values $\left(k_{p}, k_{d}\right)$ with improved GA techniques. PD is widely used in industrial applications and its algorithm can be described as:

$$
M(t)=-k_{p} \theta_{h u b}(t)-k_{d} \frac{d \theta_{h u b}(t)}{d t}
$$

where $\theta_{h u b}(t)$ is the hub angle measured at the output

$M(t)$ is the control signal which defines the input torque to the system

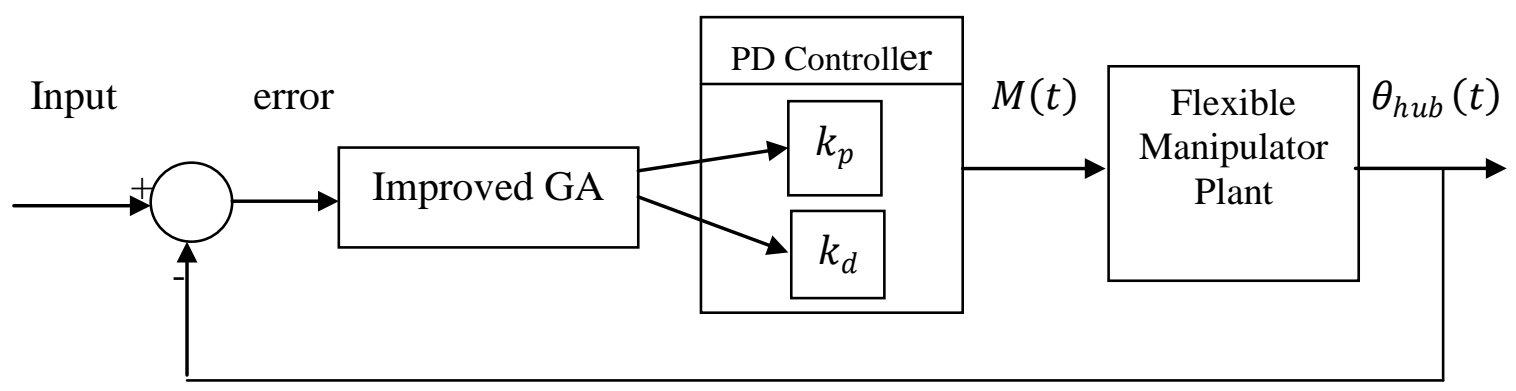

Fig. 2. Schematic of auto tuning PD controller based on improved GA

Negative feedback adds stiffness and damping effect to the system and therefore the negative signs are put in the controller equation with $\left(k_{p}, k_{d}\right)$ resulting in positive values.

\section{Simulation Results and Discussion}

Numerical simulations were carried out using MATLAB to verify the effectiveness of the proposed controller. In order to obtain more persuasive results, the plant is simulated with a 5 elements Finite Element model.

The robot arm starts with an initial tip displacement $w_{t i p}=0.01 \mathrm{~m}$ and an initial hub angle $\theta_{\text {hub }}=\frac{\pi}{2}$. The objective is to force the arm to reach setpoints $w_{\text {tip }}=$ zero and $\theta_{\text {hub }}=$ zero in the least possible time and resulting in precise positioning and minimal oscillations. This is achieved by obtaining optimal values of the feedback gains of the PD controller using GA. A population size $N_{\text {pop }}=50$ and a maximum number of generations $G_{\max }=30$ are used. A crossover probability $P_{c}=0.9$ is used for both algorithms while the probability of mutation for the SGA is $P_{m}=0.05$ and for the AGA is $P_{m}=0.05+\left(0.12 *\left(1-f_{\text {avg }}\right)\right)$. Also, the mechanical properties of the flexible arm are shown in Table 1.

Several experiments were carried out comparing Simple Genetic Algorithm (SGA) and Adaptive Genetic Algorithm (AGA) for each defined objective function. The hub angle and the tip deflection were recorded and analyzed for each case. Then, a comparison between the three objective functions is presented. The feedback gains $\left(k_{p}, k_{d}\right)$, the minimized objective function value and the time at which $\theta_{\text {hub }}$ reaches $2 \%$ of its final setpoint which is defined as $T_{2 \%}$ were all recorded.

AGA has shown more consistency over SGA where it reached global minimum almost every time. Moreover, AGA has shown faster convergence over SGA as shown in Fig. 3. In (a) 
AGA converges to the optimal solution in a fewer number of generations while in (b) SGA gets stuck at a sub-optimal solution and never converges to the global minimum.

To verify the accuracy of the obtained solutions using GA, a direct evaluation of the objective functions is done. A "map" is performed mapping a single input $\left(k_{p}, k_{d}\right)$ pair into a corresponding value of the objective function. An extensive calculation is done for several $\left(k_{p}, k_{d}\right)$ in the bounds of the feedback gains where $k_{p} \in\left(K_{p-\min }, K_{p-\max }\right)$ and $k_{d} \in$ $\left(K_{d-\min }, K_{d-\max }\right)$. The map of the objective function values is plotted and observed for each objective function. In each case, the function has shown several minima within the given range but with only one global minimum which is the same obtained by the GA.

Table 1. Mechanical Properties of the Flexible Manipulator

\begin{tabular}{l|l}
\hline \multicolumn{1}{c|}{ Parameter (Units) } & \multicolumn{1}{c}{ Value } \\
\hline \hline Length $(\mathrm{m})$ & 1.0 \\
\hline Width $(\mathrm{m})$ & 0.02 \\
\hline Thickness $(\mathrm{m})$ & 0.001 \\
\hline Material & Steel \\
\hline Density $\left(\mathrm{kg} / \mathrm{m}^{3}\right)$ & 7800 \\
\hline Modulus of elasticity $(\mathrm{Pa})$ & $207 \times 10^{9}$ \\
\hline Second moment of area $\left(\mathrm{m}^{4}\right)$ & $1.6667 \times 10^{-12}$ \\
\hline \hline
\end{tabular}

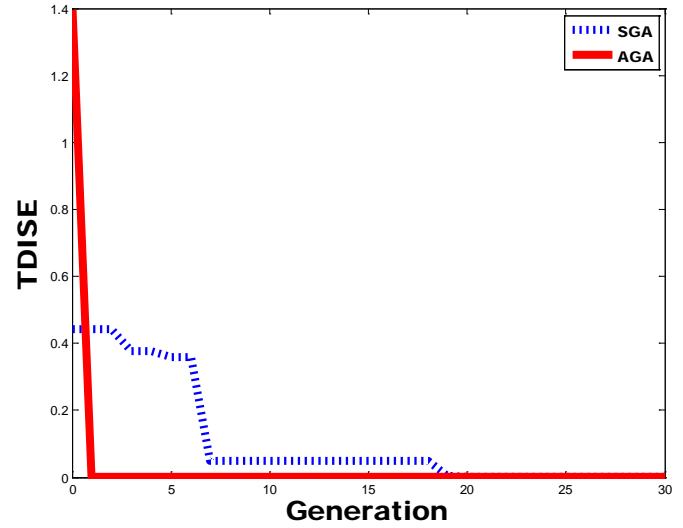

(a)

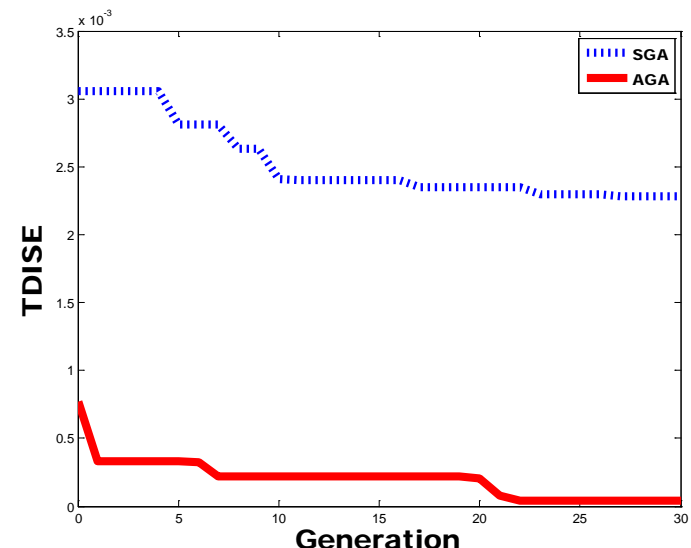

(b)

Fig. 3. Two samples of the convergence of the TDISE function to the optimal solution over 30 generations comparing both SGA (blue dotted-line) and AGA (red solid-line)

\subsection{Comparison between HAISE, TDISE and ADISE}

For each objective function, AGA has resulted in a solution which has given the best system behavior. These three solutions are compared and analyzed: $\left(k_{p}=0.6111, k_{d}=0.3277\right)$ for HAISE, $\left(k_{p}=0.0135, k_{d}=0.0849\right)$ for TDISE and $\left(k_{p}=0.0182, k_{d}=0.0446\right)$ for ADISE. As shown in Fig. 4 and Table 2, TDISE is the slowest regarding hub angle positioning with $T_{2 \%}=24$ while both HAISE and ADISE converge in similar times with $T_{2 \%}=11$. A major improvement in vibration damping results from both TDISE and ADISE as shown in Fig. 5. They appear as a fine line compared to the high oscillations given by HAISE. Fig. 6 shows a scaled figure of the tip deflection comparing both TDISE and ADISE. From Table 2, ADISE showed improvement in vibration reduction by $\sim 60 \%$ in amplitude over 
TDISE at time $=10$ seconds. Therefore, ADISE using AGA has given the best results for both angular position control and vibration control resulting in the optimal feedback gain values of $k_{p}=0.0182$ and $k_{d}=0.0446$.

Table 2. Performance comparison between HAISE, TDISE and ADISE

\begin{tabular}{l|c|c}
\hline Objective function & $T_{2 \%}$ & Tip Deflection at time=10 seconds \\
\hline \hline HAISE & 11 & 0.0813 \\
\hline TDISE & 24 & $4.76 \times 10^{-4}$ \\
\hline ADISE & 11 & $1.86 \times 10^{-4}$ \\
\hline
\end{tabular}

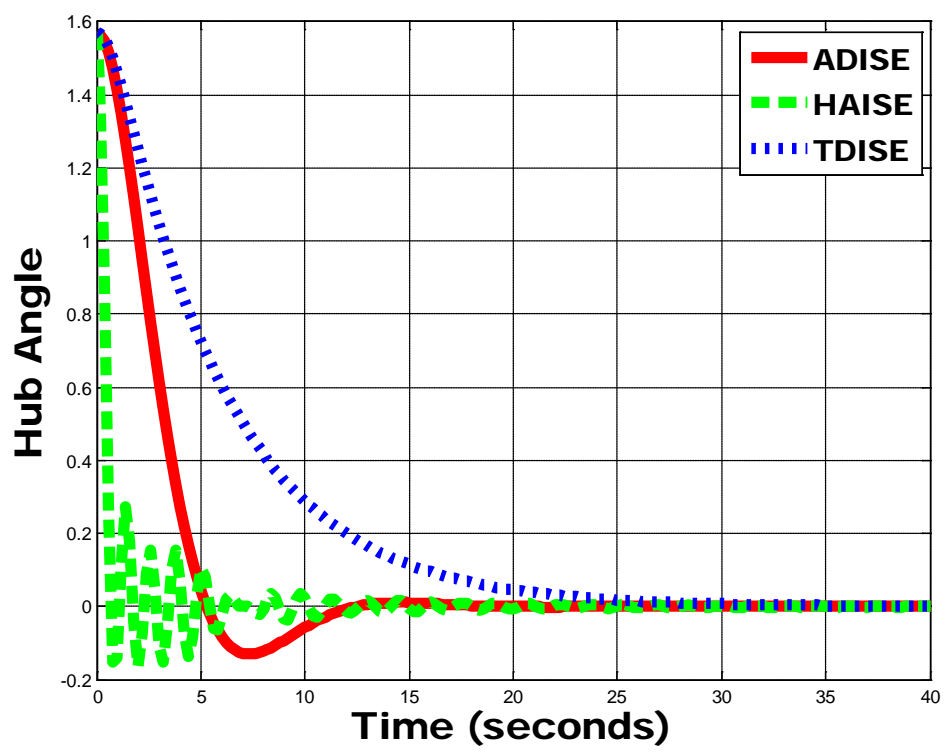

Fig. 4. The Hub Angle over time for the best solutions of HAISE shown in green dashedline, TDISE shown in blue dotted-line and ADISE shown in red solid-line

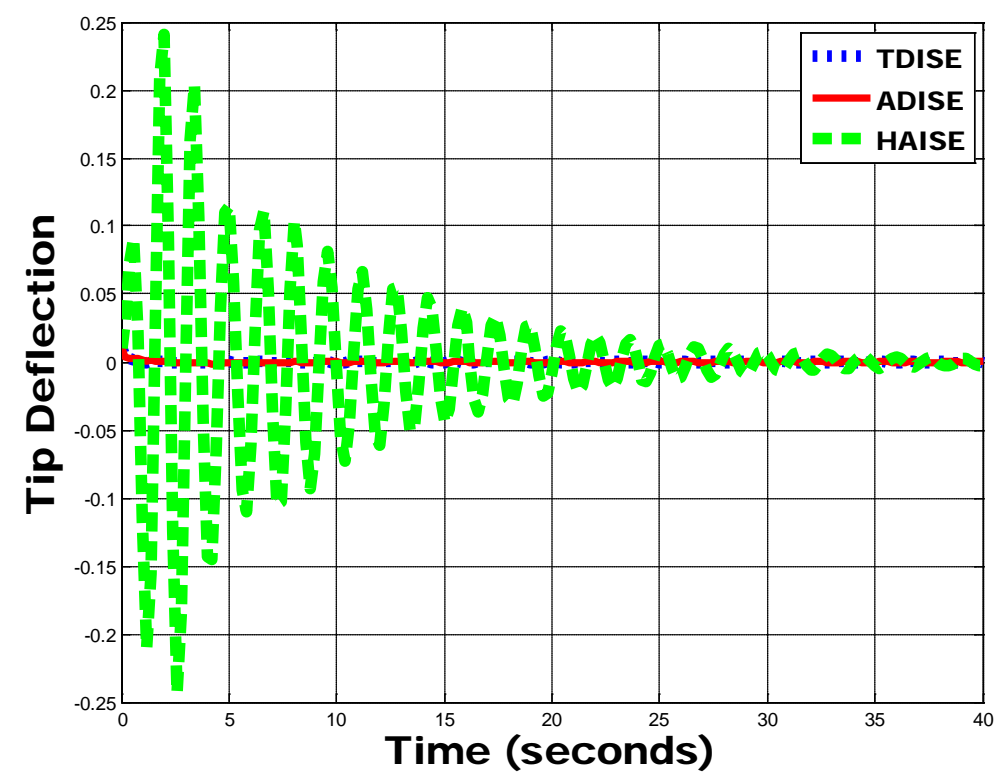

Fig. 5. The Tip Deflection over time for the best solutions of HAISE shown in green dashed-line, TDISE shown in blue dotted-line and ADISE shown in red solid-line 


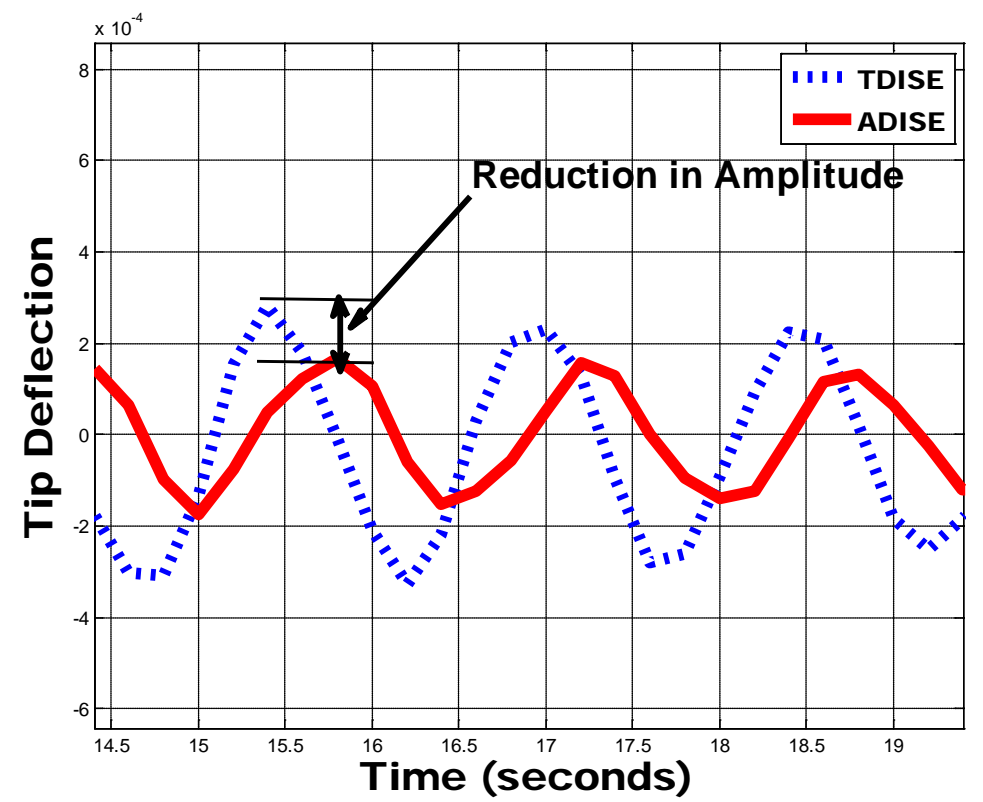

Fig. 6. The Tip Deflection over time comparing TDISE and ADISE

\section{Conclusion}

A new proposed genetic algorithm technique has been used successfully to achieve optimal angular position and optimal tip motion performance by tuning the feedback gains of the PD controller for a single-link flexible manipulator. A new adaptive mutation operator and three different objective functions have been used and compared. Results obtained using GA has been compared with direct evaluations of the objective function to verify the accuracy of the proposed technique. This paper has also included results of simulation experiments demonstrating the effectiveness of the proposed GA approach. AGA showed better results over SGA where it converged to the optimal solution in fewer generations and it rarely gets stuck at a local minimum. The combination of AGA with the concept of multi-objective optimization greatly improved the system response resulting in precise angular positioning and minimal oscillations of the flexible link.

Future work may include applying AGA and the concept of multi-objective optimization in the position and vibration control of a wide range of systems such as multiple link rigid robots and multiple link flexible robots.

\section{References}

[1] S.K. Dwivedy and P. Eberhard, "Dynamic Analysis of Flexible Manipulators, A Literature Review”, “Mechanism and Machine Theory, Elsevier”, Vol.41, March 2006, pp. 749-777.

[2] M.Benosman and G.Le.Vey, "Control of Flexible Manipulators: A Survey”," Robotica”, vol.22, pp. 533-545.

[3] R.H.Cannon Jr., E. Schmitz, "Initial Experiments on the End-Point Control of a Flexible One-Link Robot”, “The International Journal of Robotics Research”, Vol.3, No.3, 1984, pp. 62-75.

[4] V. Feliu, K.S.Rattan and H.B.Brown Jr., "Adaptive Control of a Single-Link Flexible Manipulator”, “IEEE Control Systems Magazine”, Vol.10, No.2, 1990, pp. 29-33. 
[5] P.T.Kotnik, S.Yurkovich and U.Ozguner, "Acceleration Feedback for Control of a Flexible Manipulator Arm”, “Journal of Robotic Systems”, Vol.5, No.3, 1988, pp. 181196

[6] S.S.Ge, T.H.Lee and G.Zhu., "Genetic Algorithm Tuning of Lyapunov-Based Controllers: An Application to a Single-Link Flexible Robot System", "IEEE Transactions on Industrial Electronics”, Vol.43, No.5, 1996.

[7] K.Takahashi and M.Sasaki, "Remarks on Tip Angular Position Control of a Single-Link Flexible Robot Arm Using Modified Lyapunov Function", "Proceedings of the 2010 IEEE International Conference on Mechatronics and Automation”, 2010.

[8] I.A.Mahmood, S.O.R.Moheimani and B.Bhikkaji, "Precise Tip Positioning of a Flexible Manipulator Using Resonant Control”, “IEEE/ASME Transactions on Mechatronics”, Vol.13, No.2, 2008.

[9] Y.P.Chen and H.T.Hsu, "Regulation and Vibration Control of an FEM-Based SingleLink Flexible Arm Using Sliding-Mode Theory”, "Journal of Vibration and Control”, Vol.7, No.5, 2001, pp. 741-752.

[10] E.Pereira, S.S. Aphale, V.Feliu and S.O.Moheimani, "Integral Resonant Control for Vibration Damping and Precise Tip-Positioning of a Single-Link Flexible Manipulator", “IEEE/ASME Transactions on Mechatronics”, Vol.16, No.2, 2011.

[11] M.Baroudi, M.Saad, W.Ghie, A.Kaddouri and H.Ziade, "Vibration Controllability and Observability of a Single-Link Flexible Manipulator", " $7^{\text {th }}$ International MultiConference on Systems Signals and Devices, IEEE”, 2010.

[12] V.G.Moudgal and W.A.Kwong, "Fuzzy Learning Control for a Flexible-Link Robot”, "IEEE Transactions on Fuzzy Systems", Vol.3, No.2, 1995.

[13] Z.Su and K.Khorasani, "A Neural-Network-Based Controller for a Single-Link Flexible Manipulator Using the Inverse Dynamics Approach”, “IEEE Transactions on Industrial Electronics”, Vol.48, No.6, 2001.

[14] R.J.Wai and M.C.Lee, "Intelligent Optimal Control of Single-Link Flexible Robot Arm”, “IEEE Transactions on Industrial Electronics”, Vol.51, No.1, 2004.

[15] D.Goldberg, Genetic Algorithms in Search, Optimization and Machine Learning, Addison-Wesley, 1989.

[16] B.Zain, M.O.Tokhi and S.F.Toha, "PID-Based Control of a Single-Link Flexible Manipulator in Vertical Motion with Genetic Optimisation”, “Third UKSim European Symposium on Computer Modeling and Simulation, IEEE”, 2009.

[17] M.Srinivas and L.M.Patnaik, "Adaptive Probabilities of Crossover and Mutation in Genetic Algorithms”, “IEEE Transactions on Systems, Man and Cybernetics”, Vol.24, No.4, April 1994.

[18] YAN Tai-shan, “Adaptive Genetic Algorithm Simulating Human Reproduction Mode and Its Application in Multi-Peak Function Optimization”, “International Workshop on Intelligent Systems and Applications, IEEE”, 2009

[19] M.Tawfik, "Dynamics and Stability of Stepped Gun Barrels with Moving Bullets", "Advances in Acoustics and Vibrations, Hindawi", 2008

[20] J.H.Holland, Adaptation in natural and articial systems, Ann Arbor : The University of Michigan Press, 1975.

[21] K.F.Man, K.S.Tang and S.Kwong, "Genetic Algorithms: Concepts and Applications", “IEEE Transactions on Industrial Electronics”, Vol.43, No.5, Oct. 1996, pp. 519-534.

[22] S.N.Sivanadam and S.N.Deepa, Introduction to Genetic Algorithms, Springer, 2008.

[23] R.L.Haupt and S.E.Haupt, Practical Genetic Algorithms, John Wiley \& Sons, 2004. 